\title{
Grey Correlation Analysis of the Bending Resistance of Lightweight Thermal Insulation Composite Wall
}

\author{
Song Liu ${ }^{1}$, Yuze Tian ${ }^{1}$, a, Jihong Liu ${ }^{1}$,Wang Chen ${ }^{1}$,Tianhuan Wang ${ }^{1}$ and \\ Junzhe $\mathrm{Li}^{1}$ \\ ${ }^{1}$ University of science and technology Liaoning, Anshan City, Liaoning Province, China. \\ atianyuzhe@163.com
}

Keywords: concrete; composite wall; bending resistance; grey correlation

Abstract: The research of light thermal insulation composite wall has great significance to the study of alleviating the energy shortage situation and promoting the sustainable development of China. In this paper, the gray correlation analysis was carried out to determine the indicators that influence the bending resistance, including the thickness of the light steel keel, the thickness of the fiber cement plate and the foaming ratio of the concrete. The results of the gray correlation analysis are consistent with the test results of the wall, which indicates that the method of grey correlation analysis can be used to analyze the bending resistance of thermal insulation composite wall, which has a strong practicality.

\section{Introduction}

Nowadays, 95\% new buildings of China belong to high-energy consumption buildings, and energy consumption has gradually become a bottleneck to the sustainable development of economy in our country, so in face of the lack of energy in our country, it is urgent for us to research building energy-saving $^{[1]}$. Cast-in-place lightweight thermal insulation composite wall is a new, energy saving and environmental protection wall materia ${ }^{[2]}$.The basic structure of it is the light steel keel frame, both sides are covered with fiber cement flat framework, and the hollow part is poured with tail slag and other industrial wastes as the main raw materials, the new lightweight rigid insulation materials made by chemical foaming. The utility model has the advantages of light weight, high strength and good thermal insulation effect, and solves the defects of large energy consumption and pollution of the environment of the traditional materials, and meets the high requirements of the national standard of energy saving.

The basic structure of cast-in-place lightweight thermal insulation composite wall has light steel keel frame, with both sides covered with fiber cement pressure plate, the hollow part using the special machinery and equipment for on-site mixing, and slurry fillilng by chemical foam, and the surface formed by direct decoration. The different characteristics of the keel, the pressure plate and the slurry have different effects on the properties of the composite wall. In this paper, a large number of experiments were carried out to analyze the relationship between the thermal insulation composite wall and the influence of different parameters on the bending resistance of the wall.

\section{Index System of bending properties of thermal insulation composite wall}

The basic structure of cast-in-place lightweight thermal insulation composite wall has light steel keel frame, with both sides covered with fiber cement pressure plate, the hollow part using the special machinery and equipment for on-site mixing, and slurry filling by chemical foam, and the surface formed by direct decoration. The above three indexes are selected as the main content of the flexural performance evaluation.

1.1 Evaluation index system of thermal insulation composite wall bending properties

(i) The thickness of the light steel keel: The light steel keel structure is through the synergism of light steel keel and the engineering board.Keel spacing is generally $400-600 \mathrm{~mm}$, and keel thickness rangs from 0.4 to $2.0 \mathrm{~mm}$. As the main structure of the load, the keel has certain influence on the bending performance of the wall; 
(ii) The thickness of the fiber cement plate: The main materials are fiber, cement, silica fume and other additional materials, a special production process, cement plate, after pulping, papermaking, compressor pressure, million tons of production and four maintenance procedures of the building, has been widely used in various fields of the construction industry because of its superior performance.Fiber cement pressure plate thickness ranges between $2.5-90 \mathrm{~mm}$;

(iii) The foaming ratio of the concrete: The concrete slurry is a kind of foam insulation material, which forms a closed foam hole in the concrete, and makes the concrete lightweight and thermal insulation. The foaming ratio of concrete slurry is 3-6 times.

1.2 Selection of bending properties index of thermal insulation composite wall

Table 1 Evaluation parameters of The Thermal Insulation Composite Wall Bending Properties

\begin{tabular}{cccc}
\hline evaluation index & Wall 1 & Wall2 & Wall 3 \\
\hline keel thickness $(\mathrm{mm})$ & 0.6 & 0.4 & 0.4 \\
\hline fiber cement pressure plate thickness $(\mathrm{mm})$ & 10 & 12 & 8 \\
\hline foam multiple & 5 & 4 & 6 \\
\hline
\end{tabular}

To determine the weighing values for assessment index of insulation composite wall bending performance

Weight is the relative value of the importance of factors. In this paper, the weight of the index is determined by consulting the expert opinions.

According to the index system selected above, through investigation and expert advice, with reference to previous thermal insulation composite wall bending performance analysis where indicators are given different values ${ }^{[3]}$ and the importance analysis ${ }^{[4]}$, this paper determines the relative importance of each index. The establishment of index pairwise judgment matrix, the calculation of the maximum eigenvalue and check of the consistency of judgment matrix ( Table 2), the verification results show that the consistency of the matrix is satisfactory, through the AHP method, the keel, fiber cement pressure plate, foam multiple accounted for weight were $0.47,0.32,0.21$ respectively.

\section{Grey correlation analysis of thermal insulation composite wall bending properties}

\subsection{Grey correlation analysis of evaluation index}

The gray system theory takes the system that "some information is known, some information is unknown" as the research object. Thermal insulation composite wall bending properties analysis uses the grey correlation analysis established by professor Deng Julong ${ }^{[5-6]}$ Each gray correlation degree of the model in the grey relational differences information space and the bull 's-eye is,called bull's-eyes proximity, short for bull's-eyes degree. By comparing the parameters of the target distance between the size of the target, the standard of measuring the effect of the parameters is determined. Gray relational analysis is an important part of gray system theory. It can reveal the relationship between factors in the system by quantitative description, and judge the primary and secondary relations of the factors.

Grey relational analysis steps are as follows:

(i) The establishment of a data sample matrix. Thermal insulation composite wall bending properties analysis consists of three parameters analysis scheme set $\mathrm{X}$, and three indicators to compose index set A, So the sample effect matrix of the solution set

$$
X=\left[\begin{array}{ccc}
0.6 & 0.4 & 0.4 \\
10 & 12 & 8 \\
5 & 4 & 6
\end{array}\right]
$$

(ii) The establishment of a decision matrix 
Three factors are the factors of affecting the seismic performance of thermal insulation composite wall. The parameters are normalized and range standardization method is used to analyze raw data processing. The standardization of evaluation index set can be divided into two types. The standardization of $A=\left\{A_{1}, A_{2}, A_{3}\right\}$ evaluation index set can be divided into two types.

(a) For efficiency index, hope the samples value "bigger is better", then

$$
r_{i j}=\frac{x_{i j}-\min _{i j}}{\max _{1 \leq i \leq m}-\min _{i \leq m} x_{i j}},(1 \leq i \leq m ; 1 \leq j \leq n)
$$

(b) For type cost index, hope sample values "as small as possible" , then

$$
r_{i j}=\frac{\max _{1 \leq i \leq m} x_{i j}-x_{i j}}{{\max x_{i j}-\min x_{i j}}_{1 \leq i \leq m}},(1 \leq i \leq m ; 1 \leq j \leq n)
$$

Standardization is calculated by range transformation matrix,

$R=\left(r_{i j}\right)_{n \times m}=\left(r_{1}, r_{2}, r_{3}, \cdots, r_{m}\right)$

Among them, $r_{i}(i=1,2,3)$ This is the effect of the solution vector (as table).

Table 2 Normalized decision matrix

\begin{tabular}{cccc}
\hline evaluation index & Wall1 & Wall 2 & Wall3 \\
\hline Keel thickness $(\mathrm{mm})$ & 1 & 0.66 & 0.66 \\
\hline Fiber cement pressureplate thickness $(\mathrm{mm})$ & 0.83 & 1 & 0.66 \\
\hline Foam multiple & 0.83 & 0.66 & 1 \\
\hline
\end{tabular}

(iii) The bull 's-eye distance calculation

The proximity of the actual situation of the wall and distance to target, determines whether the thermal insulation composite wall bending properties are good or bad. The optimal effect vector is selected to define as the bull 's-eye. Set the bull 's-eye $r=(0.6,12,6)$, Standardized matrix $r=(1,1,1)$.Comparing the evaluation value and the distance. Size between the bull 's-eye of three walls could determine the thermal insulation composite wall bending properties. By the bull 's-eye distance calculation formula of calculating different parameters and the distance between the effect of the optimal vector:

$$
E=\left|r_{i}-r\right|=\sqrt{W_{1}\left(r_{i 1}-r_{1}^{0}\right)^{2}+w_{2}\left(r_{i 2}-r_{2}^{0}\right)^{2}+\cdots+w_{n}\left(r_{i n}-r_{n}^{0}\right)^{2}}
$$

The bull 's-eye distance calculation results are shown in table 3. The smaller the effect of vector distance to target, the better the performance of composite wall bending, and on the other hand, the bending performance is poor.

Table3 Target distance calculation results

\begin{tabular}{cccc}
\hline & Wall 1 & Wall 2 & Wall 3 \\
\hline Target & 0.124 & 0.280 & 0.302 \\
\hline
\end{tabular}

3.2Grey correlation analysis results of thermal insulation composite wall bending properties According to the results of gray correlation analysis shown in Table 3, the bending performance of the wall is sorted, and the order from good to bad is: wall 1, wall 2, wall 3 . The experimental results of flexural capacity are: the wall body 1 surface cracks when vertically loaded with $6.000 \mathrm{KN} / \mathrm{m} 2$; wall 2 surface cracks when vertically loaded with $5.012 \mathrm{KN} / \mathrm{m} 2$; wall 3 surface cracks when vertically loaded with $4.893 \mathrm{KN} / \mathrm{m} 2$. Analysis results are basically consistent with the experimental results. 


\section{Conclusion}

Grey correlation analysis system of lightweight thermal insulation is a complex grey system. By the method of grey correlation analysis, we can obtain objective quantitative evaluation and analysis of bending performance of thermal insulation composite wall. Analysis results are consistent with the experimental situation, which shows that the method is practicable. The method can be used to qualitatively analyze the flexural properties of the composite wall, which is of great significance to the study of the flexural properties of the composite wall.

\section{Acknowledgements}

This work was financially supported by Liaoning Provincial Department of education project "- light assembly type light steel wall construction complete technology development and application (2016HZZD06)"; University of Science and Technology Liaoning students' innovation and entrepreneurship training program of steel lightweight wall seismic performance research ".

\section{References}

[1] Wang Xiaowen, Liu Yisheng. Systems Engineering-Theory\&Practice, 2012, 34(09) : 2274- 2282.

[2] Liu Daixi, Tian Yuze, Xiao Dan, Pan Changsheng. Journal of Anshan University of Science and Technology, 2015, 38(03) : 178- 180, 204.

[3] Li Li, Wang Lijiu. Journal of Dalian University of Technology, 2011, 51(04) : 599- 603.

[4] Li Weijun, Ye Fei. Journal of Systems Engineering and Electronics, 2001, 23(2) : 55-57.

[5] Deng Julong, in: Gray control system. Huazhong University of Science and Technology Press.

[6] Deng Julong, in: Gray system basic method. Huazhong University of Science and Technology Press. 